\title{
Compact DNA Droplet Concentration Detection System Based on Impedance Measurement
}

\author{
Ji-Seong Park, ${ }^{1,2}$ Mi-So Lee, ${ }^{1,2}$ Chan-Young Park, ${ }^{1,2}$ \\ Yu-Seop Kim, ${ }^{1,2}$ Hye-Jeong Song, ${ }^{1,2}$ and Jong-Dae Kim ${ }^{1,2 *}$ \\ ${ }^{1}$ Department of Convergence Software, Hallym University, Chuncheon 24252, Republic of Korea \\ ${ }^{2}$ Bio-IT Research Center, Chuncheon 24252, Republic of Korea
}

(Received August 1, 2019; accepted April 2, 2020)

Keywords: impedance, label-free, droplet, concentration, Chlamydia trachomatis DNA

We propose a label-free system that estimated the concentration of DNA droplets in sub-microliter volume based on the impedance measurement method. A USB oscilloscope with a built-in function generator was employed to reduce the system size instead of existing lock-in amplifiers or network analyzers. An electrode pair was fabricated with patterns on a printed circuit board (PCB). To minimize the measurement variation of the impedance between experiments, the electrode was made hydrophilic and the periphery of the electrode was blocked with a hydrophobic substance to prevent the droplet from spreading. For this purpose, the hydrophilicities of gold, tin, and silver coatings available in the PCB fabrication process and the hydrophobicities of various materials were investigated to select the most hydrophilic material to be used for the electrode coating. The proposed system was evaluated with various concentrations of saline solution and Chlamydia trachomatis DNA. Multiple measurements for a day experiment were performed for several days to investigate the precision of the proposed system. The measurement coefficient of variation (CV) of the proposed system was less than $3.7 \%$ for saline solution and $1.8 \%$ for DNA solution, showing that the proposed system is sufficiently precise.

\section{Introduction}

While most biosensors that detect biomolecules use fluorescent labeling techniques for sensitive and specific detection, the label-free technology offers advantages such as the simplification of analysis procedures and the reusability of samples. ${ }^{(1-11)}$ Label-free methods using the electrical properties of biochemicals are very promising biosensing methods because the detection limit is very low and the completion time is relatively short. ${ }^{(6,10,12)}$ Typical applications are electrochemical impedance spectroscopy (EIS), which measures impedance changes according to the concentration or amount of biochemicals, and electric cell-substrate impedance spectroscopy (ECIS), which is used to estimate micromotions and responses to drugs in cells or to evaluate the barrier function of cancer or stem cells. ${ }^{(3,4,13-22)}$ On the other

*Corresponding author: e-mail: kimjd@hallym.ac.kr https://doi.org/10.18494/SAM.2020.2806 
hand, ECIS can be regarded as a kind of EIS by using the phenomenon that the impedance between electrodes increases with cell mobility.

All of these electrical sensors require electrodes, and most are built on complementary metal-oxide-semiconductor (CMOS), glass, and plastic substrates. ${ }^{(4,9,15,16,20,22-26)}$ However, the lack of stable facilities for the production of these electrodes hinders the practical use of impedance sensing. In most studies, lock-in amplifiers, frequency and network analyzers, and LCR meters are used as impedance analyzers, but they take up much space and are difficult to control. This problem also prevents the wide use of impedance sensing methods. ${ }^{(4,6,8,12,27)}$ A variety of high-performance USB oscilloscopes with built-in function generators can be an alternative to space-saving equipment.

To minimize the amount of the DNA sample loaded on the electrode for impedance measurement, it is advantageous to make the target sample into a droplet form of sub-microliter volume. However, if the shape of the droplet formed on the electrode is not uniform, the impedance change measured per experiment may be large. Therefore, for a stable impedance measurement, the shape of the droplet that forms on the electrode should be constant each time. We have reported properties for electrodes of various materials and shapes. ${ }^{(28)}$ Through this study, it was found that it is important to make the electrode hydrophilic and make the periphery of the electrode hydrophobic so that the droplet always has a constant shape.

In this paper, we propose a system to measure the concentration of DNA droplets in sub-microliter volume using a compact USB oscilloscope and an electrode fabricated by a stable printed circuit board (PCB) manufacturing method. To construct the hydrophilic electrode with the hydrophobic periphery, the degree of hydrophilicity or hydrophobicity of the materials available in the PCB manufacturing process was investigated. Because the PCB manufacturing material was not sufficiently hydrophobic, various tapes were also tested to construct hydrophobic peripheries.

The proposed system was verified using various concentrations of saline and DNA solutions. Experimental and daily fluctuations of the proposed system were investigated by repeated measurements over several days. Experimental results show that the proposed system is sufficiently precise for measuring the concentration of DNA droplets.

\section{Materials and Methods}

\subsection{Overall system}

Figure 1 shows the biochemical reagent measurement system proposed in this paper. The sine wave voltage generated by the function generator of the USB oscilloscope (Analog Discovery 2, Digilent, USA) adopted in this system is converted into current by the input resistance $\mathrm{R}$ of the inverting amplifier and flows to the electrode connected to the feedback. The impedance of the solution exposed to the electrode can be determined from the applied voltage (chA) measured using the oscilloscope and the output (chB) of the amplifier and the reference resistance $\mathrm{R}$. The oscilloscope is connected to the PC via a USB interface and controlled by a Python program developed using a software development kit provided by a USB oscilloscope vendor to sample the input and output voltages of the amplifier. 
USB

oscilloscope

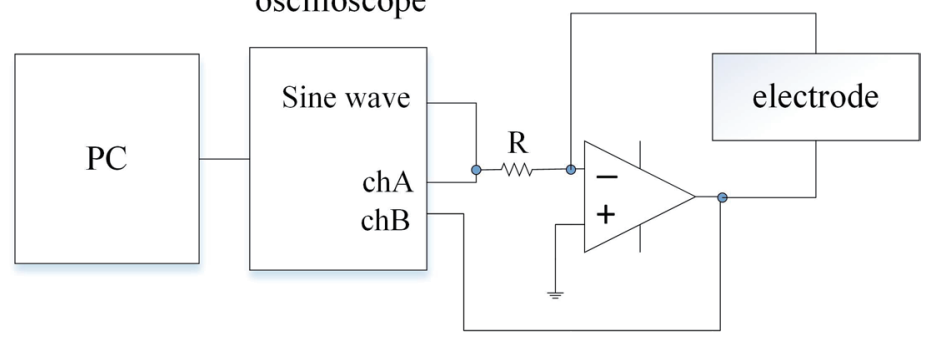

Fig. 1. (Color online) Overall system block diagram.

In the inverting amplifier structure shown in Fig. 1, the gain of the amplifier is easily obtained because it is expressed as the ratio of the reference resistance to the solution impedance. That is, the ratio of the output voltage of the amplifier to the voltage applied to the amplifier, that is, the complex gain, is multiplied by the reference resistance to obtain the impedance of the solution. The complex gain can be obtained from the two discretized trigonometric functions after the acquisition of the input and output voltages. The complex gain can be calculated by a lock-in detection method and fitting each sine wave. The fitting method has a disadvantage in that the amount of calculation is larger than that in the lock-in detection method generally used, but it is reported that the fitting method is stable when the amount of noise is more than a certain amount. ${ }^{(29)}$ Therefore, the fitting method is applied in this paper.

Figure 2 shows the details of connections of the amplifier and electrode. Since the impedance range of the target solution varies depending on the application, various reference resistances were prepared to broaden the application range. One of the eight reference resistors can be selected by setting the general-purpose output ports of the USB oscilloscope, which are connected to the selection pins of the analog multiplexer. The capacitor ' $\mathrm{Cs}$ ', which is connected in parallel with the electrode, is necessary to stabilize the amplifier and essential for high-precision operational amplifiers.

Figure 3 shows the graphical user interface of the measurement system. The edit boxes labeled 'Freq' and 'Ref' are used to enter the frequency and reference resistance values used in the measurement. After loading the sample on the electrode and pressing the 'measure' button, the real, imaginary, and absolute values of the measured impedance will appear in the edit boxes labeled 'real', 'imag', and 'abs', respectively. The 'graph' button shows the obtained sine functions to verify that there is no problem with the system.

\subsection{Fabrication of electrodes}

The shape of the droplets loaded onto the electrodes should be consistent for a stable impedance measurement. The hydrophilic surface and hydrophobic periphery of the electrode guaranteed this when the loaded reagent amount was consistent. Since the electrodes were fabricated by the stabilized PCB process, various materials for the surface and periphery were 


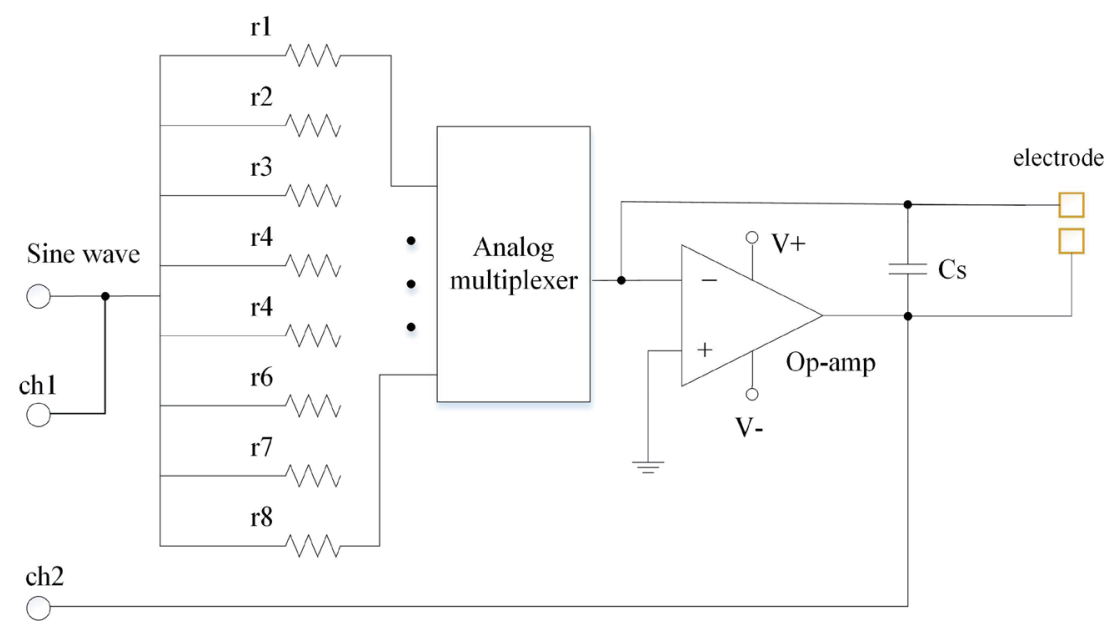

Fig. 2. (Color online) Detailed diagram of the inverting amplifier and electrode.

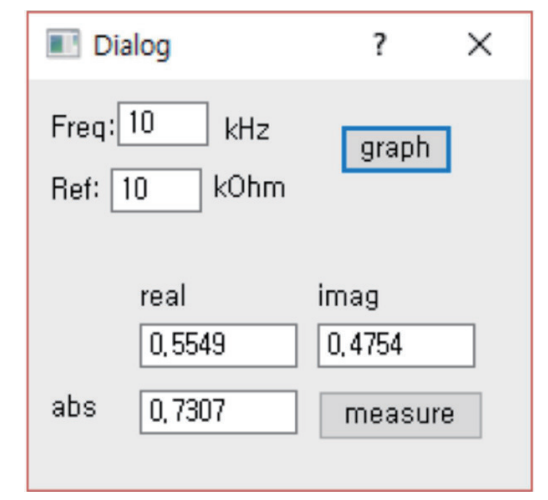

Fig. 3. (Color online) Graphical user interface.

tested first among the materials used in the PCB process. Gold, silver, and tin coatings were investigated in terms of their suitability to hydrophilic surfaces, and PCB substrates and solder masks were tested for the hydrophobic periphery. The shape of the droplets formed on the surface of each material was examined and qualitatively analyzed. Figure 4 shows the shape of the droplets on various surfaces. Three photographs from the left show the shapes of the droplets on the hydrophilic surfaces. The droplet on the gold coating was the most widespread as shown in the leftmost picture in Fig. 4. Therefore, in this study, electrodes were coated with gold. A PCB substrate or a solder mask could be used as a hydrophobic material to prevent the droplet from spreading. The right two figures in Fig. 4 are the results, and it can be seen that the droplet is more agglomerated on the PCB substrate surface than on the solder mask. In other words, the PCB substrate surface is more hydrophobic than the solder mask.

The PCB electrodes shown in Fig. 5 were fabricated using these materials to prevent the spreading of droplets. Figure 5(a) shows a structure that prevents spreading with the solder 


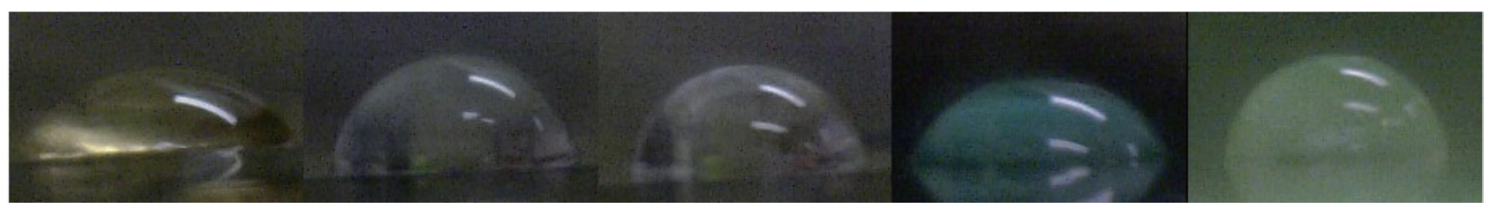

Fig. 4. (Color online) Droplets on various surfaces (from left: gold, tin, silver, solder mask, and PCB substrate).

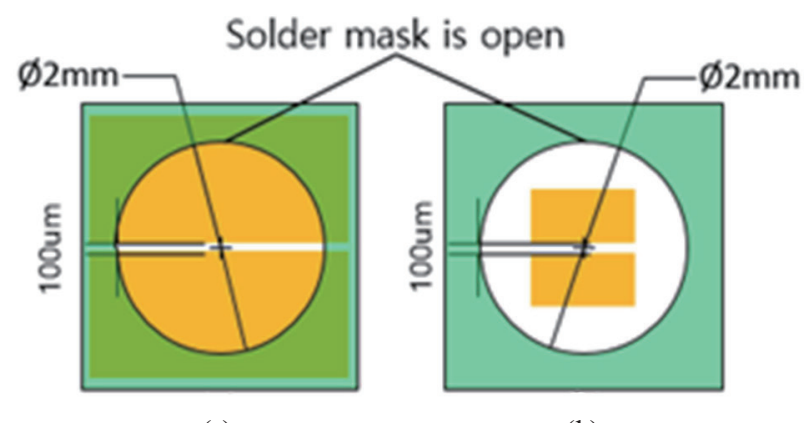

(a)

(b)

Fig. 5. (Color online) Structure of electrodes to prevent spreading of droplets using PCB process materials.

mask, and Fig. 5(b) shows a structure that prevents spreading with the PCB substrate. Note that the solder mask is located above the electrode and that the PCB substrate is below the electrode. Experimental results showed that the solder mask was insufficient to prevent spreading and that the droplet on the PCB substrate randomly flew down because the substrate was lower than the electrode. As a result, neither of these methods reliably prevented the spread of the droplet. Therefore, the solder mask was opened to expose all the areas of the electrode, and the degree of spread of the droplet was observed by attaching various tapes punched with $2 \mathrm{~mm}$ holes, and the measurement variations were examined. As a result, the easily available electrical insulating tape and waterproof bandage showed the best performance. In this study, a relatively inexpensive electrical insulating tape was used. Figure 6 shows the implementation of the electrode and analog front end.

\subsection{Experiments}

In this paper, we used saline solution to verify the performance of the proposed system. To measure the impedance of each reagent concentration, saline was prepared at five concentrations, namely, $0.625,1.25,2.5,5$, and $10 \mathrm{mg} / \mathrm{ml}$. Before loading the droplets, the electrode surface was cleaned with tissue (Kimwipes, Yuhan-Kimberly, Korea). In one experiment, the concentration of each reagent was measured ten times to investigate the experimental variation. This experimental process was repeated once daily for 8 days to estimate the daily variation of the measured values. 


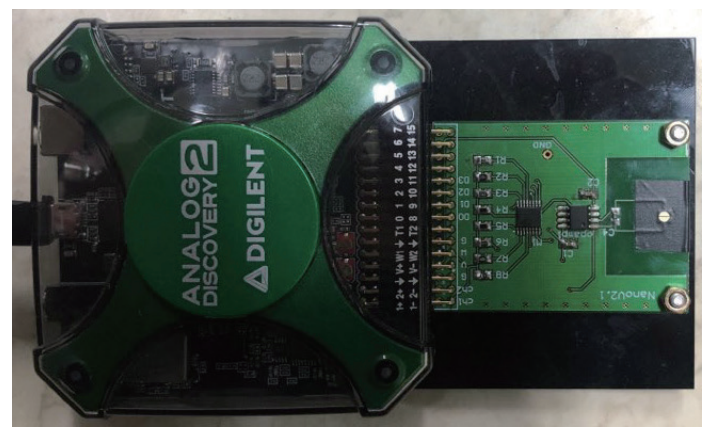

Fig. 6. (Color online) Implementation of the proposed system.

We verified the ability to measure DNA concentration using Chlamydia trachomatis (CT) DNA. Similar to saline solutions, DNA solutions were also prepared for five concentrations, namely, 2.5, 5, 10, 25, and $50 \mathrm{ng} / \mu \mathrm{l}$. As the saline test results showed that the experimental variance was very small, measurements were performed five times for each concentration in the DNA experiment. The experiment was repeated for four days to estimate daily fluctuation. In all experiments, the reference resistance was $64 \mathrm{k} \Omega$ and the stimulation frequency was fixed at $100 \mathrm{kHz}$.

\section{Result and Discussion}

Figure 7 shows the distilled water (DW) droplet dropped three times and then photographed. Unlike the electrodes constructed in our previous study, the shape of the droplet was very constant. $^{(28)}$

Table 1 shows the coefficient of variation (CV) of the impedances of the saline solution measured 10 times in each experiment. The CV of all experiments was less than $3.7 \%$ and was found to be very stable. Table 2 shows the daily variation of the mean value for each concentration and is shown as a graph in Fig. 8. When the saline concentration was low and the impedance was large, the CV was relatively small. However, it was found that the smaller the impedance, the larger the CV. These results imply that it is important to determine the reference resistance to suit the impedance range of the reagent depending on the application. However, the very small CV in the same day experiment means that calibration can reduce the measurement error by as much as the experimental variation shown in Table 1. In other words, it means that error can be reduced by performing the measurement after calibration using a reference reagent. Also, since the impedance and concentration are in a very linear relationship as shown in Fig. 8, the two-point calibration alone will be enough for the concentration estimation of the saline solution.

Overall, the CV of CT DNA was lower than that in the case of saline, as shown in Table 3 (maximum $1.8 \%$ vs $3.7 \%$ ). This result was inferred to be due to the impedance of the DNA solution being larger than that of the saline solution (1.3-14.8 k $\Omega$ vs $0.3-2.2 \mathrm{k} \Omega$ ). However, 


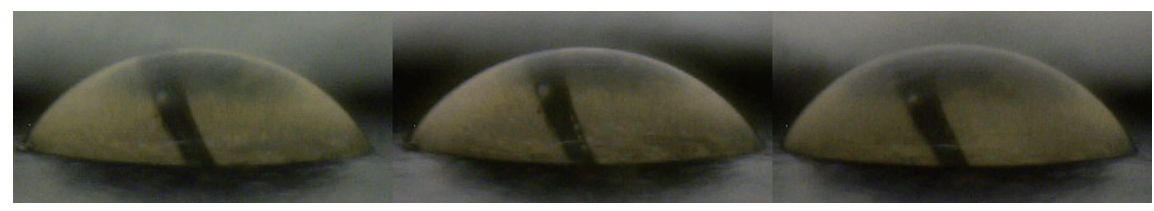

Fig. 7. (Color online) DW droplets loaded on the proposed electrode consecutively.

Table 1

Experimental variation of $\mathrm{CV}$ of measurement for saline solution (\%).

\begin{tabular}{lccccc}
\hline Concentration $(\mathrm{mg} / \mathrm{ml})$ & 1st day & 2nd day & 3rd day & 6th day & 8th day \\
\hline 0.625 & 0.9 & 1.0 & 1.0 & 0.7 & 0.7 \\
1.2 & 1.9 & 0.8 & 1.2 & 2.0 & 1.0 \\
2.5 & 2.1 & 3.7 & 1.9 & 2.2 & 1.2 \\
5 & 1.3 & 1.4 & 0.5 & 1.5 & 2.2 \\
10 & 1.1 & 2.3 & 2.7 & 3.2 & 2.1 \\
\hline
\end{tabular}

Table 2

Daily variation of average impedance of saline solution $(\mathrm{k} \Omega)$.

\begin{tabular}{lcccccccc}
\hline Concentration $(\mathrm{mg} / \mathrm{ml})$ & 1st day & 2nd day & 3rd day & 6th day & 8th day & Mean & SD & CV (\%) \\
\hline 0.625 & 2.1 & 2.1 & 2.0 & 2.2 & 2.1 & 2.1 & 0.07 & 3.2 \\
1.2 & 1.2 & 1.2 & 1.1 & 1.2 & 1.2 & 1.2 & 0.04 & 3.6 \\
2.5 & 0.8 & 0.8 & 0.7 & 0.8 & 0.8 & 0.8 & 0.06 & 7.1 \\
5 & 0.5 & 0.5 & 0.4 & 0.6 & 0.5 & 0.5 & 0.06 & 10.8 \\
10 & 0.4 & 0.3 & 0.3 & 0.3 & 0.4 & 0.3 & 0.05 & 14.1 \\
\hline
\end{tabular}

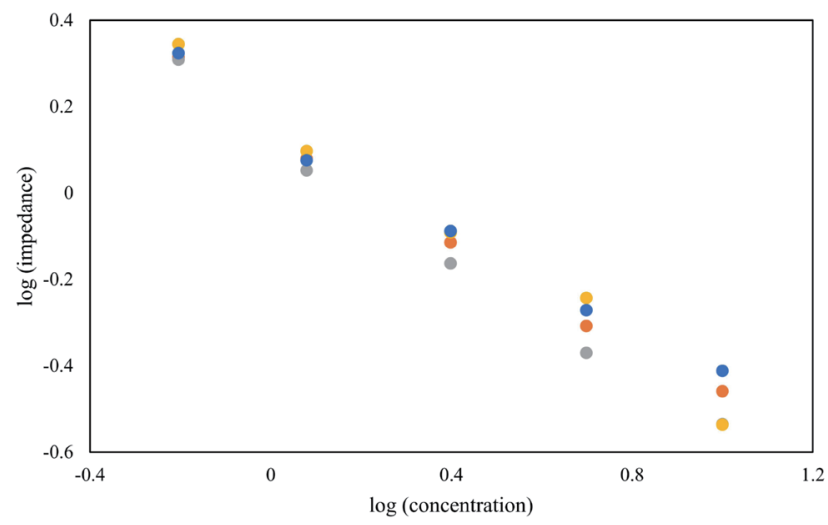

Fig. 8. (Color online) Relationship between concentration and impedance of saline solution (data points of the same color are the average of the measurements of the same day).

Table 3

Daily variation of $\mathrm{CV}$ of measurement for CT DNA (\%).

\begin{tabular}{lcccc}
\hline Concentration $(\mathrm{ng} / \mu \mathrm{l})$ & 1st day & 2nd day & 3rd day & 4th day \\
\hline 2.5 & 1.7 & 1.5 & 1.5 & 1.5 \\
5 & 0.8 & 1.7 & 1.8 & 1.5 \\
10 & 0.3 & 0.7 & 0.8 & 0.9 \\
25 & 1.0 & 1.1 & 0.8 & 1.0 \\
50 & 0.5 & 1.0 & 0.5 & 0.9 \\
\hline
\end{tabular}




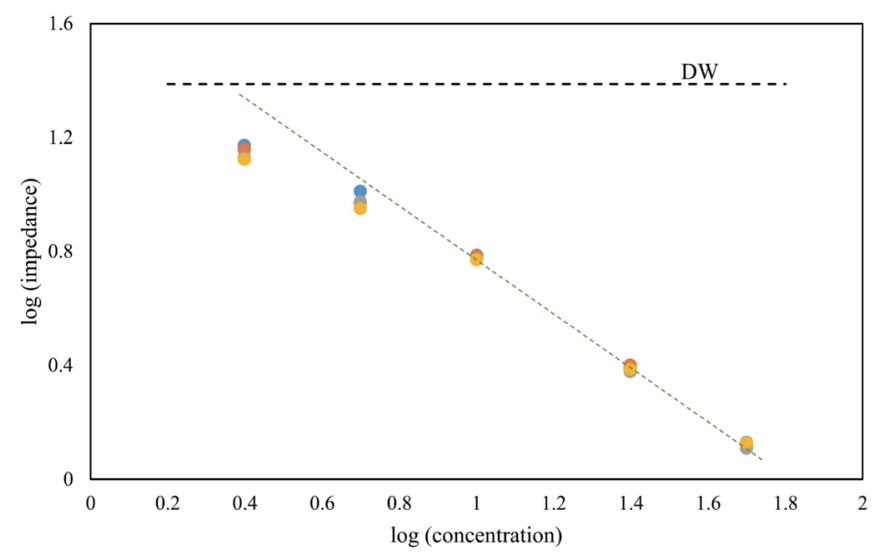

Fig. 9. (Color online) Relationship between concentration and impedance of CT DNA (data points of the same color are the average of the measurements of the same day).

Table 4

Daily variation of average impedance of CT DNA $(\mathrm{k} \Omega)$.

\begin{tabular}{lccccccc}
\hline Concentration $(\mathrm{ng} / \mu \mathrm{l})$ & 1st day & 2nd day & 3rd day & 4th day & Mean & SD & CV $(\%)$ \\
\hline 2.5 & 14.8 & 14.4 & 13.5 & 13.3 & 14.0 & 0.74 & 5.3 \\
5 & 10.2 & 9.4 & 9.4 & 8.9 & 9.5 & 0.55 & 5.8 \\
10 & 6.1 & 6.1 & 5.9 & 5.9 & 6.0 & 0.10 & 1.7 \\
25 & 2.5 & 2.5 & 2.4 & 2.4 & 2.4 & 0.05 & 2.0 \\
50 & 1.3 & 1.3 & 1.3 & 1.3 & 1.3 & 0.03 & 2.2 \\
\hline
\end{tabular}

since the $\mathrm{CV}$ of $3.7 \%$ was observed regardless of the solution, it was found that the proposed system can accurately measure the impedance. In Fig. 9, the horizontal dashed line represents the impedance of the DW droplet. When the concentration is high, that is, the impedance is relatively small, the impedance changes linearly with the concentration (ref. tilted dashed line). However, when the concentration gradually decreased, it converged to the DW impedance. Therefore, it is advisable to use several nonlinear methods to predict the concentration of the DNA solution. In the case of DNA, the daily variation of CV was relatively small (maximum 5.8\%), as shown in Table 4; therefore, it may be used without calibration according to the application.

\section{Conclusions}

In this paper, we proposed a compact DNA droplet concentration measurement system using a USB oscilloscope and a PCB electrode. The introduction of the USB oscilloscope has greatly reduced the size of the impedance measuring device. We also proposed an electrode capable of stable loading of droplets. For this purpose, the best hydrophilic electrode was selected from the PCB electrode coatings, and the droplets were prevented from spreading around by attaching the hydrophobic tape with a hole onto the electrode. The shape of the droplet loaded on the proposed electrode was very constant, and the variation of the measured impedance was very small. Experimental and daily variations of saline and DNA solutions were investigated 
to verify the proposed system. The experimental results show that the proposed system can be used for precision measurements of the reagent concentration.

The proposed system is a system for estimating the concentration by measuring the impedance of a small amount of solution and is suitable for applications where the amount of measurement reagent is critical. Also, it is advantageous when a portable device is needed, because the system is compact and can be connected to a standard computing device through a standard interface.

\section{Acknowledgments}

This research was supported by Government-wide R\&D Fund project for infectious disease research, HG18C0012.

\section{References}

1 A. Biela, M. Watkinson, U. C. Meier, D. Baker, G. Giovannoni, C. R. Becer, and S. Krause: Biosens. Bioelectron. 68 (2015) 660. https://doi.org/10.1016/j.bios.2015.01.060

2 C. Iliescu, D. P. Poenar, and S. T. Selvan: J. Micromech. Microeng. 20 (2009) 022001. https://doi. org/10.1088/0960-1317/20/2/022001

3 I. O. K'Owino and O. A. Sadik: Electroanalysis 17 (2005) 2101. https://doi.org/10.1002/elan.200503371

4 H. Ma, R. W. Wallbank, R. Chaji, J. Li, Y. Suzuki, C. Jiggins, and A. Nathan: Sci. Rep. 3 (2013) 2730. https:// doi.org/10.1038/srep02730

5 R. McGuinness: Current Opin. Pharmacol. 7 (2007) 535. https://doi.org/10.1016/j.coph.2007.08.004

6 P.-D. Nguyen, T. B. Tran, D. T. X. Nguyen, and J. Min: Sens. Actuators, B 197 (2014) 314. https://doi. org/10.1016/j.snb.2014.02.089

7 A. Oseev, M.-P. Schmidt, S. Hirsch, A. Brose, and B. Schmidt: Sens. Actuators, B 239 (2017) 1213. https://doi. org/10.1016/j.snb.2016.09.118

8 T. Schwarzenberger, P. Wolf, M. Brischwein, R. Kleinhans, F. Demmel, A. Lechner, B. Becker, and B. Wolf: Physiol. Meas. 32 (2011) 977. https://doi.org/10.1088/0967-3334/32/7/S18

9 M. Shariati: Biosens. Bioelectron. 105 (2018) 58. https://doi.org/10.1016/j.bios.2018.01.022

10 R. Sharma, S. E. Deacon, D. Nowak, S. George, M. Szymonik, A. Tang, D. Tomlinson, A. Davies, M. McPherson, and C. Wälti: Biosens. Bioelectron. 80 (2016) 607. https://doi.org/10.1016/j.bios.2016.02.028

11 G. Ventimiglia and S. Petralia: J. Bionanosci. 3 (2013) 428.

12 Z. Zou, S. Lee, and C. H. Ahn: IEEE Sens. J. 8 (2008) 527. https://doi.org/10.1109/JSEN.2008.918907

13 M. Angstmann, I. Brinkmann, K. Bieback, D. Breitkreutz, and C. Maercker: Cytotherapy 13 (2011) 1074. https://doi.org/10.3109/14653249.2011.584863

14 C. Berggren, B. Bjarnason, and G. Johansson: Biosens. Bioelectron. 13 (1998) 1061. https://doi.org/10.1016/ S0956-5663(98)00058-X

15 C.-M. Chen and M. S.-C. Lu: 2017 19th Int. Conf. Solid-State Sensors, Actuators and Microsystems (TRANSDUCERS) (2017) 1632.

16 G. Chornokur, S. K. Arya, C. Phelan, R. Tanner, and S. Bhansali: J. Sens. 2011 (2011) 983752.

17 T. M. Curtis, M. W. Widder, L. M. Brennan, S. J. Schwager, W. H. van der Schalie, J. Fey, and N. Salazar: Lab Chip 9 (2009) 2176. https://doi.org/10.1039/b901314h

18 R. Ehret, W. Baumann, M. Brischwein, A. Schwinde, and B. Wolf: Med. Biol. Eng. Comput. 36 (1998) 365. https://doi.org/10.1007/BF02522485

19 D. Holmes, D. Pettigrew, C. H. Reccius, J. D. Gwyer, C. van Berkel, J. Holloway, D. E. Davies, and H. Morgan: Lab Chip 9 (2009) 2881. https://doi.org/10.1039/b910053a

20 T. B. Tran, C. Baek, and J. Min: PLOS ONE 11 (2016) e0153813. https://doi.org/10.1371/journal.pone.0153813

21 T. B. Tran, P. D. Nguyen, C. Baek, and J. Min: Biosens. Bioelectron. 77 (2016) 631. https://doi.org/10.1016/ j.bios.2015.10.030

22 Y. Zhou, S. Basu, E. Laue, and A. A. Seshia: Biosens. Bioelectron. 81 (2016) 249. https://doi.org/10.1016/ j.bios.2016.02.069 
23 I. Giaever and C. R. Keese: Nature 366 (1993) 591. https://doi.org/10.1038/366591a0

24 A. Manickam, A. Chevalier, M. McDermott, A. D. Ellington, and A. Hassibi: 2010 IEEE Int. Solid-State Circuits Conf. (ISSCC) (2010) 130.

25 A. Mucha: 2012 PhD Thesis. Technische Universität München.

26 R. C. Nordberg, J. Zhang, E. H. Griffith, M. W. Frank, B. Starly, and E. G. Loboa: Stem Cells Transl. Med. 6 (2017) 502. https://doi.org/10.5966/sctm.2015-0404

27 R. Sinville and S. A. Soper: J. Sep. Sci. 30 (2007) 1714. https://doi.org/10.1002/jssc.200700150

28 C.-Y. Park, M.-S. Lee, Y.-S. Kim, H.-J. Song, and J.-D. Kim: Sens. Mater. 31 (2019) 245. https://doi. org/10.18494/SAM.2019.2089

29 C.-Y. Park, M.-S. Lee, Y.-S. Kim, H.-J. Song, and J.-D. Kim: Sens. Mater. 30 (2018) 1499. https://doi. org/10.18494/SAM.2018.1831 